This is a penultimate draft of a paper forthcoming in the South African Journal of Philosophy.

\title{
A Neglected Response to the Paradoxes of Confirmation
}

\begin{abstract}
Hempel's paradox of the ravens, and his take on it, are meant to be understood as being restricted to situations where we have no additional background information. According to him, in the absence of any such information, observations of FGs confirm the hypothesis that all Fs are $G$. In this paper I argue against this principle by way of considering two other paradoxes of confirmation, Goodman's 'grue' paradox and the 'tacking' (or 'irrelevant conjunct') paradox. What these paradoxes reveal, I argue, is that a presumption of causal realism is required to ground any confirmation; but once we grant causal realism, we have no reason to accept the central principles giving rise to the paradoxes.
\end{abstract}

\section{Hempel's paradox of the ravens}

Here's an initial — though, as we shall see, simplistic — outline of Hempel's (1945) paradox of the ravens. The paradox involves two compelling principles, SUPPORT and EQUIVALENCE, and two hypotheses, [Rave] and [NoB], which are logically equivalent:

\author{
SUPPORT \\ A generalization is confirmed (supported) by observations of its instances- \\ so long as no counterinstances have been observed. (I.e. observations of \\ $F G$ s confirm the hypothesis 'All $F$ s are $G$ ', so long as no $F$ s that are not $G$ \\ have been observed.)
}

\section{EQUIVALENCE}

Evidence that confirms a hypothesis equally confirms any logically equivalent hypothesis.

[Rave] All ravens are black.

[NoB] All non-black things are non-ravens.

Very briefly: by SUPPORT, observation of a red pencil, say, being an observation of a nonblack non-raven, confirms hypothesis [NoB], and thus, by EQUIVALENCE, also confirms the logically equivalent hypothesis [Rave] that all ravens are black. It is at least prima facie paradoxical that a red pencil should come out positively supporting a hypothesis about ravens!

Hempel bites the bullet, however, and endorses that conclusion. The reason we think it paradoxical, he claims, is that we mistakenly think that [Rave] is making a claim just about ravens; but the fact that [Rave] is equivalent to $[\mathrm{NoB}]$ shows that this is simply not so. ${ }^{1}$ Indeed, once we see that [Rave] is also equivalent to the hypothesis [Noth] that nothing is a non-black raven, we realize that [Rave] is in fact making a claim about every object in the world. Thus, even observations of black non-ravens, e.g. black shoes, come out confirming

\footnotetext{
${ }^{1}$ I suspect some readers will question the claim that [Rave] and [NoB] are logically equivalentsurely the first is about ravens whereas the second is about non-black things? It may be easier to see the equivalence once one sees, what is indicated in the very next sentence of the main text, that each hypothesis entails and is entailed by the hypothesis [Noth] that nothing is a non-black raven.
} 
[Rave] - by virtue of its being equivalent to [Noth]. In short, no observation is neutral (or irrelevant) to [Rave] — any observation will either support [Rave] or refute it!

But, it is important to note that Hempel takes these conclusions to hold only in contexts where there is no further background knowledge or assumptions. He gives the following example to show how SUPPORT can be nullified by the presence of additional information. Suppose someone attempted to test the hypothesis [SodS]: 'All sodium salts burn yellow', by checking whether a yellow flame was produced when a piece of pure ice was held to it. They observed that a yellow flame was not produced and presented this result as confirming the hypothesis, 'Whatever does not burn yellow is no sodium salt', and then, by appeal to EQUIVALENCE, as confirming the original hypothesis, [SodS]. Hempel acknowledges that this would strike us as paradoxical. He notes that the situation is different if we had done the same test with an as yet unidentified substance, found that a yellow flame was not produced, and then, on subsequent analysis, found that the substance contained no sodium salt. In this case, we would take the result of the test to confirm [SodS], even if it had turned out that the substance was pure ice. What gives? Here is Hempel's answer:

\begin{abstract}
Now the only difference between the two situations here considered is that in the first case we are told beforehand the test substance is ice, and we happen to "know anyhow" that ice contains no sodium salt; this has the consequence that the outcome of the flame-colour test becomes entirely irrelevant for the confirmation of the hypothesis and thus can yield no new evidence for us. (Hempel 1945, p. 19).
\end{abstract}

Thus, Hempel appears to hold that background information can render observation irrelevant to a hypothesis even though the same observation in the absence of that background information would confirm that hypothesis. So e.g. the view that the observation of a red pencil confirms [Rave] only seems paradoxical, on this view, because we already know that it is a red pencil, and so, not a raven; if we knew merely that the observed object was a nonblack non-raven, then the observation would indeed confirm [Rave] by way of confirming [NoB].

Here are three further examples which show that SUPPORT is vulnerable to background knowledge.

Example 1. Suppose we come across a white crow in the arctic. According to SUPPORT, that observation, being an observation of a non-black non-raven, confirms [Rave]. But, since crows and ravens are biologically related species, this observation of a white crow surely renders it less likely, in other words, disconfirms, the hypothesis that all ravens are black-in which case, SUPPORT is violated. ${ }^{2}$ The background information which does the damage here is the relatedness of crows and ravens.

Example 2 (from Good 1967). First, suppose we have come to the conclusion that one of the following two hypotheses about our world is true:

(H1) The world contains a hundred black ravens, no non-black ravens, an a million other birds.

\footnotetext{
${ }^{2}$ Another casualty is the view that any observation will either confirm or refute a generalization: clearly, disconfirmation here is not to be understood as outright refutation.
} 
(H2) The world contains a thousand black ravens, one white raven, and a million other birds. [So, on this hypothesis, not all ravens are black.]

Now, suppose we are examining birds to see if [Rave] is correct and come across a black raven. This observation is more likely under hypothesis $(\mathrm{H} 2)$ than hypothesis $(\mathrm{H} 1)$, so the observation confirms (H2), on which [Rave] is false, and disconfirms (H1), on which [Rave] is true! Again, the example clearly relies on the presence of additional background information-whatever it is which renders (H1) and (H2) the only alternatives.

Example 3 (from Sainsbury 1988). Consider the following generalization:

[Snake] All snakes inhabit countries other than Ireland.

The more snakes we observe in other countries, thriving in a variety of environments, the less likely it is that there are no snakes in Ireland. Thus, a collection of observations of instances of [Snake] could disconfirm rather than confirm [Snake]! In this case it is the information we accrue, as we come across more and more instances, which eventually undermines SUPPORT.

So, it is well known that background information may render SUPPORT invalid. But Hempel, and, along with him, many commentators, have generally taken the following, qualified version of SUPPORT to be untarnished by the sorts of examples we have considered:

\section{Qualified-SUPPORT (Q-SUPPORT)}

In the absence of further background information (beliefs, assumptions), a generalization is confirmed by observations of its instances. ${ }^{3}$

No doubt Q-SUPPORT is intuitively compelling at first blush; but, as we'll see, it is plain false! I will make my case by way of considering two other paradoxes of confirmation: Goodman's (1955) 'grue' paradox and the so-called 'tacking' or 'irrelevnt conjunct'paradox. What these paradoxes reveal, I will argue, is that it is in fact only in the presence of a background presumption of causal realism that observing an $F$ to be $G$ may support the generalization that all $F$ s are $G$. It is striking that leading figures writing on confirmation theory-Carnap, Hempel, Goodman, and Quine, for example - have, under the influence of logical positivism, simply denied that there is any mind-independent causal oomph (or necessitation) in the world: the regularities in the world are, for them, merely contingent and ungrounded; no events were 'made to' happen; there is no underlying reason why things happen as they do. These philosophers accordingly failed to mention the realist blocking of the paradoxes I am going to present here. The central contention of this paper is that (inductive) confirmation in general rests on the presumption of causal realism, and that such a commitment to causal realism, albeit tacit and undefended, undermines the support principles underpinning the paradoxes of confirmation.

\section{The 'grue' paradox}

Goodman's (1955) 'new riddle of induction' as he calls it arises from SUPPORT alone. ${ }^{4}$ Let 'grue' be a predicate that applies to anything that has been observed before now and found to

\footnotetext{
${ }^{3}$ The earlier qualification 'so long as no counterinstances have been observed' is not needed, since any such observations would count as part of the background information.
} 
be green, and to anything else, i.e. anything that has not been observed before now, just in case it is blue. Suppose that all emeralds that have been observed up until now have been green. By SUPPORT, these observations confirm the following hypothesis:

[Green] All emeralds are green.

But, by the definition of 'grue', it follows that the emeralds that have been observed up until now have also been grue. So, by SUPPORT, the same observations also confirm:

[Grue] All emeralds are grue.

The problem is, [Green] and [Grue] make divergent predictions about emeralds discovered in the future: the former predicts that they will be green, while the latter predicts that will be blue. We evidently favour the prediction that they will be green. But, Goodman asks, why 'project' the predicate 'green' rather than the predicate 'grue' here?

He is good enough to provide an answer: it is simply because the predicate 'green' is better entrenched in our language and theories than 'grue'. Goodman is offering us a psychological explanation of why we project 'green' rather than 'grue' here, but not a justification. Pace Hume, he does not think a justification is possible.

I won't quibble with that answer now; the pertinent point here is that, for Goodman, it is the background information we have - to the effect that the predicate 'green' is better entrenched than 'grue'-which tells in favour of [Green] over [Grue].

So, the 'grue' paradox appears to give us yet more evidence of the impact of background information on the applicability of SUPPORT. But why should we take this fact to give any succour to Q-SUPPORT? One might just as well infer (what I in fact take to be the case) that background information is actually necessary for confirmation generally. Indeed, I think a cursory consideration of the tacking paradox reveals just this.

\section{The tacking (or irrelevant conjunct) paradox, and the obvious culprit}

This paradox arises from a cousin of SUPPORT and a principle concerning what we might call the 'transmission' of confirmation:

\section{SUPPORT*}

A hypothesis is confirmed by observations of its consequences (or phenomena it predicts).

\footnotetext{
${ }^{4}$ A referee has pointed out important differences in the projects that Hempel and Goodman were engaged in: Hempel was concerned with the issue of specifying criteria for evaluating past performance whereas Goodman was concerned with the issue of grounding for future predictions. My concern here is with the second project; while Hempel may not have been addressing it, his observations certainly have implications for it - and that is how I am cashing out his paradox. In any case, the realist stance I press later applies to both projects, so the differences are immaterial for the purposes of this paper. (I thank the referee for forcing clarity on this point.) ${ }^{5}$ Quine (1969) suggests restricting SUPPORT to generalizations involving natural-kind terms. This is along the lines of Goodman's proposal, for it is tantamount to holding that observations of $F G \mathrm{~s}$ confirm All Fs are $G$ conditional on background information to the effect that $F$ and $G$ are naturalkind terms.
} 


\section{TRANS}

Observations which confirm a hypothesis, $H$, also confirm any hypothesis $H$ entails.

Now, let $O$ be any true observation report, $H$ be any hypothesis whatsoever consistent with $O$, and $H^{*}$ be the conjunction ' $O \& H$ '. By SUPPORT', $O$ confirms $H^{*}$; but, in that case, by TRANS, $O$ also confirms $H$, which is entailed by $H^{*}$. So, we get the paradoxical result that any observation that has been made supports any hypothesis that is consistent with that observation. For example, the observation that I sneezed just now comes out confirming the hypothesis that the earth will explode tomorrow!

This is the tacking paradox. ${ }^{6}$ The result is clearly absurd. However, it is easy to see what the source of the problem is. Obviously, our observation $O$ confirms (in this case, entails) the first conjunct of $H^{*}$ (reminder: $H^{*}={ }^{\top} O \& H^{\top}$ ), but it need have no bearing on the second conjunct whatsoever - for that conjunct may, as in our example, have just been 'tacked' on. We need some independent reason for thinking that support for the first conjunct will be support for the second, or for thinking that the first conjunct itself supports the second. The air of paradox disappears even if we may not know what we should replace SUPPORT* ${ }^{*}$ or TRANS with.

The 'grue' paradox is an instance of the tacking paradox

Now, returning to Goodman's paradox, [Green] and [Grue] can be seen to be equivalent to the following conjunctions, respectively:

[\&Green] $\quad{ }^{\top}$ All observed emeralds are green ${ }^{\top} \&^{\lceil}$All unobserved emeralds are green ${ }^{\top}$

[\&Grue $\quad\left\lceil\right.$ All observed emeralds are green ${ }^{\rceil} \&{ }^{\lceil}$All unobserved emeralds are blue

Our evidence, that the emeralds we have observed so far have been green, clearly supports the first conjuncts of these hypotheses, but we are owed an answer as to why it should support the second conjuncts. This effectively boils down to the question of why the truth of the first conjunct should lead us to expect the second conjunct to be true. Therefore, Goodman's paradox is a simply an instance of the tacking paradox.

Goodman is well aware of the foregoing point; he concurs that, in the absence of any other information, there is nothing to recommend [Green] over [Grue]. Without any background assumptions or information, we have the traditional problem of induction: there is nothing to recommend hypotheses about the unobserved on the basis of the observed! But, whereas Goodman settles for a Humean solution to his paradox - basically explaining why we favour [Green] - I contend that it is only a background presumption of causal realism, a belief that

\footnotetext{
${ }^{6}$ Hempel (1945, pp. 31-32) and Goodman (1955, pp. 67-68) are well aware of it, but do not draw the realist moral I am about to recommend. The problem is also discussed in connection with Popper's falsifiability principle - (if $A$ is falsifiable/refutable, then so too is the conjunction $[A \& B]$, for any $B$ ) - and for Hypothetico-Deductive and Bayesian accounts of confirmation. See e.g. Fitelson (2002), Chandler (2007), Crupi and Tentori (2010), and Sprenger (2011) for elaboration of problems and attempted solutions.
} 
there is mind-independent causal oomph (necessitation) in the world, which licences our inductive inferences. It is only the assumption that are objective reasons why observed objects have certain properties that entitle us to generalize. Thus, it is because, and only because, we have background information or beliefs to the effect that things like emeralds have properties in virtue of which they have the colour they do that observations of green emeralds confirm that all emeralds are green. ${ }^{7}$

The upshot of all this is that causal realists - i.e. just about everyone but a few academic philosophers - should have no truck with Q-SUPPORT or with SUPPORT*. Not all generalizations will be confirmed by their instances, and not all hypotheses will be confirmed by their consequences: it is only in the presence of some background causal narrative that confirmation makes sense; thus, some regularities will count as merely accidental regularities and will perforce be unconfirmable.

\section{Humean causation vs Realist causation}

But why should realists about causation have an advantage over anti-realists? After all, the latter, Goodman among them, don't deny that there is causation - it is their take on it which differs from the realists'. There is no obstacle to their having the same causal beliefs - that is, beliefs about what causes what, or about which regularities are laws or lawlike - as the realists. So, assuming the same background causal beliefs, what counts as confirming evidence for a realist should also count as confirming evidence for an anti-realist. Where, then, is the advantage in realism?

The advantage emerges when we consider the supposed direction of explanation between laws and their instantiations, if you will. For the realist, the laws are part of the explanation of why certain events occur or have the features they do; certain events make other events occur; events occur in virtue of other features of the world. Realists have good cause(!) then to take particular observations as data for establishing the (objective) causal structure of the world; as evidence of something that has a determinable impact beyond the observations which have been made so far.

By contrast, while the anti-realist may well believe in the same laws or regularities, these don't explain why particular events occur-indeed, there is no explanation of why they occur. Rather, the laws themselves, as well as all other regularities, are partly determined (and explained) by what actually occurs. Thus, anti-realists do not have good cause to take particular observations as evidence of anything that impacts on other events! So, it strikes me that anti-realists are not entitled to, and by their own lights, should not make any assumptions about what universal regularities there, or, consequently, about what causes what. ${ }^{9}$ Moreover, even if they have made such assumptions-because they can't help it, as Hume suggeststhey should realize, precisely because of their views on the direction of explanation, that these assumption do not in any way support (or give any grounds for expecting) future events

\footnotetext{
${ }^{7}$ So, in my view Hume was right to insist that induction must be founded on the relation of cause and effect, but remiss in not recognizing that a realist conception of cause is required.

${ }^{8}$ See Lewis (1973) for an influential and much discussed Humean account of causation.

${ }^{9}$ The same goes for assumptions like: the $F s$ we have observed to be $G$ are representative (are an unbiased sample) of $F$ s. The idea of 'representative' or 'biased' samples has no bite unless it is cashed out in realist terms; for the anti-realist, the above assumption is simply tantamount to the assumption that all $F$ s are $G$-which, as I have argued in the text, the anti-realist is not entitled to, indeed, ought not to, assume.
} 
to conform to them. While the assumptions may logically entail that a future event with property $F$ will also have property $G$, it is in fact irrational for them to take the assumptions as providing positive support for that eventuality. ${ }^{10}$

Hence my view that it is only on the assumption of causal realism, of an objective causal structure in the world, that confirmation makes sense.

\section{The paradox of the ravens reconsidered}

In rejecting Q-SUPPORT, causal realists obviously pull the rug from under Hempel's intended paradox. But, one might think that the paradox re-emerges for realists who allow (quite plausibly) that one's background information is, or could well be, such that observing a given raven to be black does confirm [Rave]. For, presumably in those circumstances observing a non-black thing to be a non-raven, e.g. observing a red thing to be a pencil, will also confirm [NoB], and, thence, by EQUIVALENCE, [Rave]. But isn't that the very result we found paradoxical?

But this worry is misguided. For the causal realist, when an observation of an $F G$ confirms the hypothesis that All Fs are $G$ it is never solely in virtue of the $F G$ being an instance of the generalization. The confirmation will be relative to some cause-invoking background theory. The background theory on which [Rave] is confirmed by an instance therefore need not take observation of a red pencil to confirm [NoB] by virtue of it being an instance of [NoB]; and there is no reason to expect the observation of the pencil to confirm the kind of causal theory that underpins [Rave].

The example of the white crow makes my point. The situation in which the white crow is observed may well be a situation in which observation of a black raven confirms [Rave]. But, as we have noted, the observation of the white crow still disconfirms [Rave], even though it is an instance of [NoB]. So, I deny that Hempel's paradox re-emerges for causal realists.

Of course, the traditional problem of induction remains; Humean sceptics will want to know what warrants the background commitment to causal realism. I have not attempted to address that issue - though, if push came to shove, I would probably defend it by way of inference to the best explanation. ${ }^{11}$ However, my goal was not to resolve the problem of induction; rather, it was to remind causal realists that they need not be concerned with the paradoxes of confirmation. These paradoxes were supposed to pose harder problems than Hume's problem of induction-Goodman, for example, takes them to be quite distinct: he thinks Hempel avoids the tacking paradox but not the grue paradox. What I hope to have shown is that they are in effect one and the same paradox, each stemming from a version of SUPPORT that causal realists will deny.

\footnotetext{
${ }^{10}$ The claim here is that it is irrational for the causal anti-realists to take this line. Of course, ordinary folk who have not thought on such matters will do so out of 'habit'. Causal anti-realists, presumably, should know better and be able to break the habit!

${ }^{11}$ I would also point out an absurd consequence of their stance. It is bad enough that, on their view, no events in the world have an explanation, a reason why they happened; but in the case of perception, they are further led to posit chains of events in the world running in parallel with, and presumably matching, our visual experiences as of those events occurring. That the occurrence of such widespread 'matching' chains of events should be mere coincidence - as the anti-realist would have us believestrikes me, not to mince words, as quite ludicrous.
} 
What is baffling is that this realist response should be entirely omitted in many discussions of the paradox, including major introductory texts - (to wit, check e.g Mackie 1963; Jackson 1975; Sainsbury 1988; Bird 1998; Ladyman 2002; Bortolotti 2008; Vickers 2012; and the Wikipedia entry on the 'Raven paradox'). ${ }^{12}$

\section{REFERENCES}

Bird, A. 1998: Philosophy of Science (London: UCL Press)

Bortolotti, L. 2008: Introduction to the Philosophy of Science (Polity Press)

Chandler, J., 2007, 'Solving the Tacking Problem with Contrast Classes', British Journal for the Philosophy of Science $\mathbf{5 8}$ pp. 489-502

Crupi, V. and K. Tentori, 2010, 'Irrelevant Conjunction: Statement and Solution of a New Paradox', Philosophy of Science 77 pp. 1-13.

Crupi, V. 2013: 'Confirmation', entry in the Stanford Encyclopedia of Science, at: $<$ http://plato.stanford.edu/entries/confirmation/>

Fitelson, B. 2002. 'Putting the Irrelevance Back into the Problem of Irrelevant Conjunction', Philosophy of Science 69 pp. 611-22.

Fitelson, B. and J. Hawthorne: 2009: 'How Bayesian Confirmation Theory Handles the Paradox of the Ravens', in E. Eells and J. Fetzer (eds.), Probability in Science (Chicago: Open Court).

Good, I. J. 1967: 'The White Shoe is a Red Herring', British Journal for the Philosophy of Science 17, p. 32.

Goodman, N. 1955: 'The New Riddle of Induction', in his Fact, Fiction, and Forecast (Harvard: Harvard University Press)

Hempel, C. G. 1945: 'Studies in the Logic of Confirmation' Mind 54 pp. 1-26; 97-121. Reprinted in C.G. Hempel, Aspects of Scientific Explanation and Other Essays in the Philosophy of Science (The Free Press: New York) pp. 3-51.

Horwich, P. 1982: Probability and Evidence (Cambridge: Cambridge University Press)

Jackson, F. 1975: 'Grue', Journal of Philosophy 72 pp. 113-31.

Ladyman, J. 2002: Understanding Philosophy of Science (London: Routledge)

Lewis, D. 1973: 'Causation', Journal of Philosophy 70 pp. 556-567.

Mackie, J.L. 1963: 'The Paradox of Confirmation' British Journal for the Philosophy of Science 13 pp. 265-277.

Quine, W. V. O. 1969: 'Natural Kinds' in his Ontological Relativity and Other Essays (Columbia: Columbia University Press).

Sainsbury, R. M. 1988: Paradoxes (Cambridge: Cambridge University Press)

Sprenger, J. 2011: 'Hypothetico-Deductive Confirmation', Philosophy Compass 6 pp. 497-508.

Vickers, J. 2012: 'The Problem of Induction', entry in the Stanford Encyclopedia of Philosophy, at url: <http://plato.stanford.edu/entries/induction-problem/>

${ }^{12}$ Acknowledgements. 